\title{
Selbstständigkeit in der Sozialen Arbeit
}

\section{Der Trend zum sozialen Markt und zur Geldleistung begünstigen Existenzgründungen}

\author{
Ludger Kolhoff
}

It seems to be a promising time for self-employment in the different fields of social work. Professionals deciding for such a way have to answer questions of personality and concepts they want to apply; but equally important are questions around ethics: Should difficulties and even misery be the foundation of my economic success?

L'époque semble propice au travail indépendant dans les différents champs du travail social. Les professionnels qui optent pour cette voie doivent pour cela répondre à des questions d'ordre personnel et choisir les concepts qu'ils soubaitent appliquer: mais d'autres questions, d'ordre éthique sont d'importance égale: La difficulté des autres, voire la misère peut-elle servir de terreau à mon succès économique?

Prof. Dr. Ludger Kolhoff hat Pädagogik, Elektrotechnik und Politikwissenschaft studiert, war u. a. Gründungsgeschäftsführer einer Beratungsgesellschaft und ist seit 1993 Hochschullehrer der Fachhochschule Braunschweig/ Wolfenbüttel.

E-Mail L.Kolhoff@gmx.de
Die Zeiten für die Gründung selbstständiger Existenzen in den Tätigkeitsfeldern Sozialer Arbeit scheinen gut zu sein. Fachkräfte, die diesen Weg beschreiten wollen, müssen sich neben persönlichen und konzeptionellen auch ethischen Fragen stellen: Kann die Not anderer Menschen die Grundlage meines wirtschaftlichen Erfolges sein?

Die Begriffe »Selbstständigkeit « und »Soziale Arbeit " schienen über Jahrzehnte politisch und wirtschaftlich unvereinbar zu sein, denn mit den Mitteln aus den sozialen Sicherungssystemen wurden bewusst fast ausschließlich nicht gewinnorientierte Träger finanziert. Die gemeinnützigen Spitzenverbände der freien Wohlfahrtspflege kontrollierten einen Markt, in dem sich nur wenige Selbstständige wieder fanden.

Seit einigen Jahren hat sich jedoch die Situation der sozialen Dienstleistungen auf deutscher, aber auch auf europäischer Ebene verändert. Sozioökonomische Veränderungen wie die demografische Entwicklung, Globalisierungseinflüsse, Rationalisierung durch den Einsatz von Informationstechnologien, die Folgen der deutschen Einheit haben die sozialen Sicherungssysteme zunehmend ausgedehnt und belastet, sodass der Gesetzgeber gezwungen ist, neue Parameter in die Felder der Sozialen Arbeit zu implementieren. Es sind die Paradigmenwechsel »Vom Recht zum Markt « und »Von der Sach- zur Geldleistung «.

\section{Vom Recht zum Markt}

Statt das Feld der sozialen Dienstleistungen nur rechtlich zu strukturieren, wurden seit Mitte der 1990er Jahre zunehmend marktorientierte Steuerungsansätze eingeführt, wie beispielsweise mit der Pflegeversicherung im Jahre 1995, die gewinnorientierten Trägern den Marktzugang ermöglichte. Im Rahmen der
Hartz-Reformen kamen gewinnorientierte Arbeitsvermittler und gewerbliche Personalberatungs- und Personalentwicklungsgesellschaften zum Zuge, die im Outplacement-Geschäft agieren. Mit der Einführung von Leistungsentgelten in der Kinder-, Jugend- und Sozialhilfe haben sich auch hier neue Möglichkeiten für Selbstständige eröffnet. Freiberufler und gewerbliche Träger finden sich in Einrichtungen der Benachteiligtenförderung, in Kindertagesstätten oder in Wohnheimen für Nichtsesshafte.

Zunehmend werden soziale Dienstleistungen ausgeschrieben, bei denen die kostengünstigsten Anbieter den Auftrag erhalten. Die Frage der Gewinnorientierung oder Gemeinnützigkeit verliert an Bedeutung. Der soziale Markt wird dereguliert und eröffnet Sozialarbeitern eine Chance auf Existenzgründungen.

Diese ökonomischen Veränderungen setzen gleichzeitig die öffentlichen und Non-Profit Träger unter Konkurrenzdruck. Immer mehr öffentliche Einrichtungen in der Sozialen Arbeit werden privatisiert und die großen freien Träger der Wohlfahrtspflege betreiben Outsourcing, um Kosten einzusparen. Dies betrifft nicht nur Servicebereiche wie Reinigung, Versorgung mit Essen (Catering), Facility-Management etc., sondern auch die Kernbereiche Sozialer Arbeit. Gewerbliche Träger sind, aufgrund anderer Organisations- und Vergütungsstrukturen in der Lage, preisgünstiger zu agieren als die etablierten Träger und beschäftigen dann auch » outgesourcte« Mitarbeiter der öffentlichen oder Non-Profit-Träger zu Niedriglöhnen. Hinzu kommt, dass im Betreuungs-, Beratungs-, Bildungs- und Qualifizierungsbereich zunehmend freie Träger sich auf ein Kernangebot und eine Kernmannschaft von Festangestellten konzentrieren und zusätzliche Dienstleistungen bei freiberuflich tätigen Honorarkräften einkaufen. Von ihnen kann man sich jederzeit trennen, das senkt das Risiko und sie sind preisgünstiger als Fest- 
angestellte, denn es gelten für die Vergütung keine tariflichen Regelungen wie bei Angestellten.

Dies ist für die einzelnen betroffenen Sozialarbeiterinnen und Sozialarbeiter teilweise existenzbedrohend, gesellschaftspolitisch hingegen funktional und an anderer Stelle für die neuen Selbstständigen in der Sozialen Arbeit wiederum existenzermöglichend. Selbstständige sind von der Sozialversicherungspflicht ausgenommen und können direkte und versteckte Subventionen in Anspruch nehmen (z. B. für Arbeitslosengeld-Empfän-

cherung mittlerweile an Finanzierungsgrenzen geraten ist, so wäre sie von Anfang an nicht finanzierbar gewesen, wenn das Sachleistungsprinzip alleine gegolten hätte.

An Finanzierungsgrenzen ist auch die Behindertenhilfe mit ihren hohen Wachstumsquoten geraten. Eine Dämpfung soll ab 2008 durch die Einführung Persönlicher Budgets erfolgen. Behinderte oder ihre gesetzlich bestellten Betreuer können selbst über ein Budget verfügen. Sie werden kalkulieren und statt eines großen gemeinnützigen Trägers

\section{"Ein erfolgreich Selbstständiger hat ein Gespür für Trends und ein Faible für Nischenmärkte»}

ger » Gründungszuschüsse« und günstige Konditionen in der Kranken- und Arbeitslosenversicherung), wodurch sie oftmals kostengünstiger agieren können als gemeinnützige freie Träger. Es handelt sich hier um einen Zielkonflikt, bei dem nicht alle gewinnen können, da es sich um Umverteilungen handelt oder um ein Nullsummenspiel.

Der Trend zu selbstständiger, gewinnorientierter Arbeit im sozialen Bereich wird im Zuge der Europäisierung weiter zunehmen. Spätestens wenn 2011 die volle Freizügigkeit für die 2004 beigetretenen mittel- und osteuropäischen Länder in Deutschland gegeben ist, wird es weitere noch kostengünstigere Anbieter geben, die in Konkurrenz zu den etablierten Trägern treten werden.

\section{Von der Sach- zur Geldleistung}

Mit der Einführung der Pflegeversicherung wurde ein weiterer Paradigmenwechsel in der Sozialen Arbeit eingeleitet. Statt der klassischen Sachleistung konnten nun auch Geldleistungen von den Versicherten in Anspruch genommen werden. Auch wenn diese nur 50 Prozent der Sachleistungen betrugen, wurden sie für die ambulante Pflege dennoch mehrheitlich gewählt. Hiermit wurde ein informeller Sektor der sozialen Dienstleistungen aufgebaut und finanziert, seien es Nachbarn oder Familienangehörige oder aber preiswerte illegal tätige Pflegekräfte aus Mittel- und Osteuropa. Auch wenn die Pflegeversi- vielleicht das kleine gewinnorientierte Unternehmen oder den Einzelunternehmer in Anspruch nehmen - besonders dann, wenn der Einzelunternehmer, bevor er sich selbstständig gemacht hat, bei dem großen Träger beschäftigt war und intensive Kontakte zum behinderten Menschen aufgebaut hat, um dessen Budget er jetzt mit seinem ehemaligen Arbeitgeber konkurriert. Wenn der Budgetnehmer dann feststellen sollte, dass die Betreuung des Einzelunternehmers preiswerter ist und noch Geld fürs Kino oder für die Urlaubsreise übrig bleibt, mag die Entscheidung schnell getroffen sein und es wird sich eine neues Feld für Selbstständige in der Sozialen Arbeit auftun.

Die Einführung von Geldleistungen statt Sachleistungen wird in formalisierter Form auch im Bereich des SGB III (Vermittlungs- oder Bildungsgutscheine) angewandt und auch im Rahmen des anstehenden Ausbaus von Kindergärten und Krippen (Hamburger Gutschein-Modell) diskutiert. Auch hier ist davon auszugehen, dass gewinnorientierte Träger und Einzelunternehmer von dem Wechsel von der Sach- zur Geldleistung profitieren werden.

Es öffnen sich in der Zukunft vielfältige Fenster für Möglichkeiten der Selbstständigkeit im Feld der sozialen Dienstleistungen. Für jeden einzelnen Sozialarbeiter, der diesen Weg der Selbstständigkeit einschlagen möchte, gelten gewisse Rahmenbedingungen und notwendige Kompetenzen, die kritisch überprüft werden sollten (vgl. nebenstehenden Kasten).

\section{Erfolgreiche Selbstständige in der Sozialwirtschaft}

Um in der Sozialwirtschaft erfolgreich zu sein, müssen Selbstständige kostengünstiger arbeiten als andere oder sie müssen Größenvorteile aufweisen. Erfolg versprechend ist eine umfassende Marktpräsenz, die zur Zeit jedoch insbesondere von den Verbänden wahrgenommen wird, da diese vom Kindergarten bis zum Pflegedienst sämtliche Bereiche der Wohlfahrt abdecken. Also sollten Selbstständige sich miteinander vernetzen, um synergetische Effekte zu nutzen, beispielsweise einen gemeinsamen Fuhrpark betreiben, Personal austauschen oder Gebäude teilen.

Eine weitere Erfolg versprechende Strategie für Selbstständige kann darin bestehen, die Qualitätsführerschaft zu übernehmen, eine innovative Dienstleistung anzubieten oder eine unverwechselbare Marke zu kreieren. Denkbar sind regionale Spezialisierungen und innovative, bisher noch nicht gedachte Zielgruppenspezialisierungen, wie ein Altersheim für Homosexuelle oder Wohnprojekte im Stil der 1960er-Jahre für in die Jahre gekommene »Alt-68er «.

Ein erfolgreich Selbstständiger erkennt Veränderungen frühzeitig, hat ein Faible für Nischenmärkte und Trends, kann diese schnell und kostengünstig besetzen und absichern, um im Konkurrenzkampf zu bestehen. In den Bereichen Alter und Pflege, Gesundheit und Krankheit wachsen derzeit die Arbeitsfelder für Selbstständige. Insbesondere der für Selbstständige interessante kaufkräftige und finanzstarke Teil der Seniorengeneration, die »Best Ager «, haben spezifische Erwartungen an das Altern, die frühzeitig zu erspüren sind. So findet sich beispielsweise im Wellnessbereich oder in der Kombination von Touristik und Sozialer Arbeit ein wachsendes Handlungsfeld für Selbstständige. Ein wachsendes Arbeitsfeld für Selbstständige eröffnet sich derzeit auch beim Thema Migration und Integration, wenn gefordert wird, Kindern mit Migrationshintergrund neue Chancen zu eröffnen.

\section{»Double-Bind « für Selbst- ständige in der Sozialen Arbeit}

Selbstständige sind einerseits von einer öffentlichen Subventionierung und somit von Gesetzen und Verordnungen, aber auch von einzelnen Entscheidungsträgern 
abhängig und müssen auf Veränderungen öffentlicher Träger reagieren können. Anderseits müssen Selbstständige nach den gleichen Kriterien arbeiten, wie jedes andere erwerbswirtschaftliche Unternehmen auch, wenn sie nicht in die Insolvenz gehen wollen.

Es werden somit widersprüchliche Botschaften transportiert, die so manchen Selbstständigen in eine Double-Bind-Situation bringen. Einerseits gelten die klassischen Botschaften des sozialen Sektors: »Hilf Menschen in Not", »Sei ein guter Mensch «, »Orientiere dich an den gesetzlichen Rahmenbedingungen und politischen Machtstrukturen «. Andererseits gelten die Botschaften der Erwerbswirtschaft: »Du bist deines Glückes Schmied «, »Verdiene viel Geld «, »Werde reich «. Beide Botschaften lassen sich schwer vereinbaren.

Nur in den wenigsten Fällen ist ein soziales Problem die Basis für eine Geschäftsidee, die im Sinne einer Win-winSituation zu einer Verbindung von Philanthrophie und Reichtum führt. In der

\section{Beispiel Selbstständigkeit: Organisationsentwicklung}

Meine Firma bietet partizipative Organisations- und Kommunalentwicklung auf wissenschaftlicher Grundlage an.

Im Rahmen eines Projektes zur Optimierung des Freiwilligenengagements im Bundesland Hessen (Landesehrenamtsagentur) wurde das Konzept sehr erfolgreich erprobt. Zusammen mit »pro regio « und der großzügigen Unterstützung durch die Gesellschaft für Konsum und Marktforschung, dem Institut für Demoskopie Allensbach und Sphinx-Survey konnte die Methode ihre problemlösenden Stärken zeigen.

Das Resümee meiner Tätigkeit ist sehr positiv. Ich schätze es, erfolgsorientiert und praktisch sozialarbeitswissenschaftlich tätig zu sein. Ich halte die Rechtsform der eingetragenen Genossenschaft (www.neuegenossenschaften.de) als am günstigsten für die Selbständigkeit in der Sozialen Arbeit. Diese potenziert die Stärken der Partnerinnen und Partner und optimiert das Dienstleistungsangebot. Mit der Genossenschaft kann ich Workshops für bis zu 500 Teilnehmenden anbieten. Selbständigkeit ist herausfordernd und interessant.

Uwe Schacher E-Mail Uwe.Schacher@t-online.de

Regel lassen sich beide Ebenen nicht miteinander verbinden. Der Markt fordert »Sei billiger als die anderen, wenn du eine Chance haben willst « und der soziale Sektor fleht »Erzeuge keine neuen Notlagen « und »Halte dich an die Spielregeln $«$.
Diese Double-Bind-Situation kann zum Burn-out der Selbstständigen führen. Selbstständige, die ihre Dienstleistung preiswerter anbieten müssen als andere, und die nicht bereit sind, andere auszubeuten, beuten sich selbst oft aus.

\section{Bedingungen der Selbstständigkeit in der Sozialen Arbeit}

Die Chancen für eine selbstständige berufliche Existenz mit sozialen Dienstleistungen wachsen. Wer diese Situation für sich nutzen will, sollte vier Aspekte berücksichtigen:

- Persönliche Voraussetzungen: Menschen, die sich selbstständig machen wollen, sollten ihre persönlichen Dispositionen und Voraussetzungen klären, ihre Stärken und Schwächen überprüfen und ihre Qualifikationen und individuellen Rahmenbedingungen reflektieren. Individuelle Voraussetzungen sind Ausdauer, Frustrationstoleranz, Kreativität, berufliche Erfahrungen und Kontakte, kaufmännische und unternehmerische Kompetenzen.

- Konzeptionelle Voraussetzungen: Entscheidend für eine erfolgreiche Existenzgründung ist, neben den persönlichen Voraussetzungen, die Qualität der Unternehmenskonzeption sowie die Bereitschaft, diese ständig zu optimieren. Die Geschäftsidee sollte sich - gemäß dem Motto »Der Wurm muss dem Fisch und nicht dem Angler schmecken « - am Bedarf der Kunden orientieren und diesen einen Nutzen versprechen. Selbstständige sollten sich also nicht in Methoden oder Probleme verstricken, für die es im betriebswirtschaftlichen Sinn keinen Markt gibt, sondern die Kundenbedürfnisse eruieren, um dann einen pragmatischen Weg in die Selbstständigkeit zu finden. Zu berücksichtigen ist, dass es sich beim sozialen Sektor um einen Quasi-Markt handelt: nur in Ausnahmefällen sind Leistungsempfänger und Leistungsvergüter identisch. Folglich sollten Selbstständige insbesondere die Finanzierungsebene, formale Rahmenbedingungen und die Interessen und Erwartungen der Kostenträger im Auge haben.

- Finanzielle Voraussetzungen: Die meisten Existenzgründungen scheitern an Engpässen bei der Finanzierung. Folglich sollte frühzeitig geklärt werden, welche Kosten entste- hen und welche Einnahmen realistischerweise erzielt werden können. Hierzu muss jeder Existenzgründer einen Kosten-, Umsatz-, Rentabilitäts- und Liquiditätsplan erstellen. Im Kostenplan sind die Personalkosten (Gehälter und Lohnnebenkosten), Sachgemeinkosten (Miete, Fahrzeugkosten, Bürobedarf etc.), Zinsen, Abschreibungen und die kalkulatorischen Kosten aufzuschlüsseln. Bei den kalkulatorischen Kosten handelt es sich um den Unternehmerlohn. Er dient nicht nur dazu, den Lebensunterhalt zu bestreiten, sondern auch zur Begleichung von Einkommenssteuer und Sozialversicherungsbeiträgen und sollte deshalb um die Hälfte höher liegen als der Lohn aus einer angestellten Tätigkeit. Der Umsatz- und Rentabilitätsplan dient dazu anhand einer Übersicht über die zu erwartenden Einnahmen (Umsatzplan) und Kosten (Kostenplan) zu eruieren, ob die Kosten gedeckt und ein angemessener Unternehmerlohn erzielt werden kann (Rentabilitätsplan). Im Liquiditätsplan wird geklärt, wie mögliche Liquiditätsengpässe abgedeckt werden können.

- Unternehmensform: Auf dem Hintergrund des Haftungsrisikos und steuerrechtlicher Bedingungen muss die Rechtsform der selbstständigen Tätigkeit entschieden werden. Dies kann eine Personengesellschaft, beispielsweise ein Einzelunternehmen, eine Gesellschaft bürgerlichen Rechts oder eine Partnergesellschaft sein, oder eine Kapitalgesellschaft, beispielsweise in Form einer $\mathrm{GmbH}$, insbesondere dann, wenn Risiken abzupuffern sind. Von der Wahl der Rechtsform sind formale Dinge wie Nachweise, Zulassungen und Genehmigungen abhängig, die ebenso wie die steuerrechtliche und versicherungstechnische Aspekte zu berücksichtigen sind.

Ludger Kolhoff 